Vietnam Journal of Mechanics, VAST, Vol.37, No. 1 (2015), pp. 57-70

DOI:10.15625/0866-7136/37/1/5865

\title{
A GLOBAL-LOCAL APPROACH TO THE DESIGN OF DYNAMIC VIBRATION ABSORBER FOR DAMPED INVERTED PENDULUM STRUCTURES
}

\author{
N. D. Anh ${ }^{1,2}$, N. X. Nguyen ${ }^{3, *}$ \\ ${ }^{1}$ Institute of Mechanics, Vietnam Academy of Science and Technology, Hanoi, Vietnam \\ ${ }^{2} \mathrm{VNU}$, University of Engineering and Technology, Hanoi, Vietnam \\ ${ }^{3} \mathrm{VNU}$, University of Science, Hanoi, Vietnam \\ *E-mail: nguyennx12@gmail.com \\ Received January 26, 2015
}

\begin{abstract}
In practice, an inverted pendulum can be used to model many real structures as the arms of robots, soil structures, or fluid structures. However, the study on the design of dynamic vibration absorber for inverted pendulum structures is very limited in the literature. To the best knowledge of the authors, however, there has been no study on the dynamic vibration absorber when the primary inverted pendulum structure is damped. This paper deals with the optimization problem of dynamic vibration absorber for inverted pendulum structures. Two novel findings of the present study are summarized as follows. First, the optimal parameters of dynamic vibration absorber for undamped inverted pendulum structures are given by using $H_{\infty}$ optimization. Second, the authors suggest a so-called global-local approach to determine approximate expressions for optimal parameters of a pendulum type absorber attached to a damped inverted pendulum structure. Finally, a numerical simulation is done for an example of the articulated tower in the ocean to validate the effectiveness of the results obtained in this work.
\end{abstract}

Keywords: Dynamic vibration absorber, inverted pendulum, damped structure, globallocal approach, analytical solutions.

\section{INTRODUCTION}

A passive vibration control device attached to a primary structure to reduce harmful vibration is called a dynamic vibration absorber (DVA). The first DVA was introduced by Frahm [1] in 1909 for mass-spring structures but it has no the damper element and is only useful in a narrow range of frequencies very close to the natural frequency of the DVA. In 1928, Ormondroyd and Den Hartog [2] found that a DVA containing a viscous damper was effective to an extended range of frequencies. The DVA proposed by Den

(C) 2015 Vietnam Academy of Science and Technology 
Hartog is now known as a standard model of DVA where a spring element and a viscous element are arranged in parallel.

For undamped mass-spring structures, there have been many optimization criteria given to design a DVA. Three typical optimization criteria are $H_{\infty}$ optimization, $H_{2}$ optimization, and stability maximization. The $H_{\infty}$ optimization was first proposed by Ormondroyd and Den Hartog [2] when the primary structure is subjected to harmonic excitation. The objective was to minimize the maximum amplitude magnification factor of the primary structure. Den Hartog [3] presented the optimal tuning ratio and damping ratio of a DVA by using a so-called fixed-point theory. It is noted that the DVA's optimal parameters derived by utilizing the fixed-point theory are only approximate solutions because some approximations are taken. Nishihara and Asami [4] proposed the exact solutions and compared these with the results given by Den Hartog. They found that both optimal tuning ratio and damping ratio presented by Den Hartog were very close to the exact solutions. Hence, the fixed-point theory provided a very good approximation of the exact solutions for the $H_{\infty}$ optimization in practice because the exact solution was too complicated. The $\mathrm{H}_{2}$ optimization criterion was given by Crandall and Mark [5] in 1963 when the primary structure is subjected to random excitation. The purpose was to minimize the area under the frequency response curve of the main structure. Iwata [6] and Asami [7] gave a DVA's optimal parameters according to the $H_{2}$ optimization. The stability maximization criterion and exact solutions of optimum parameters of a DVA were suggested by Yamaguchi [8] in 1988. The aim was to improve the transient vibration of the structure.

When the primary mass-spring structure is damped, it is difficult to obtained analytical solutions for the optimum parameters of a DVA. For the $H_{\infty}$ optimization,Ioi and Ikeda [9] used a numerical method to give empirical formulae for the optimum parameters of a DVA. Pennestri [10] suggested a min-max design of a DVA where a min-max objective function subjected to six constraint equations with seven unknown variables was found. In 2002, Asami et al. [11] presented a series solution for the $H_{\infty}$ optimization. Using an approximate assumption of the existence of two fixed points, Ghosh and Basu [12] presented a closed-form expression for the optimal tuning ratio of a DVA. Anh and Nguyen $[13,14]$ suggested approximate analytical solutions for optimal parameters of a DVA based on the idea of the criteria of the equivalent linearization method. For the $\mathrm{H}_{2}$ optimization, Asami et al. [11] gave an exact solution but their solution was extremely complicated so they proposed an approximate solution for practical use. In 2012, Tigli [15] provided the exact optimum parameters of a DVA for the $\mathrm{H}_{2}$ criterion in the case of velocity and approximate solutions when minimizing the displacement and acceleration of the main structure. For the stability optimization, Nishihara and Matsuhisa [16] gave the exact solution of a DVA's optimal parameters in 1997.

Conventional pendulum structures have received high interest in researches and engineering applications when considering many structures such as ropeway carriers, cranes or ships subjected to wave loading. A conventional pendulum model and optimal parameters of a DVA moving in the tangential direction were proposed by Matsuhisa et al. [17]. Viet et al. [18] investigated a DVA moving in the normal direction by using the 
effective damping approach. Viet et al. $[19,20]$ studied a DVA moving in both tangential and normal directions and two orthogonal DVAs for conventional pendulum structures.

A more general model called the inverted pendulum structure was suggested by Anh et al. [21]. The mass-spring structures and conventional pendulum structures can be obtained from the inverted pendulum model as special cases. In practice, many real structures can be modeled as inverted pendulums, for example, soil structures [22] or fluid structures [23]. The soil-structure interaction can be modeled by tension springs while in the fluid-structure interaction the torsion springs are due to buoyancy forces. Dong et al. [23] investigated the response of an articulated tower in the ocean subjected to deterministic and random wave loading. The tower was modeled as an upright rigid pendulum with a concentrated mass at the top and having one angular degree of freedom about a hinge with Coulomb damping which can be replaced approximately by an equivalent linear viscous one. Compliant platforms such as articulated towers are economically attractive for deep-water conditions because of their reduced structural weight compared to conventional platforms. The foundation of the tower does not resist lateral forces due to wind, waves, and currents. Instead, restoring moments are provided by a large buoyancy force, a set of guy-lines or a combination of both [24-27]. The use of dynamic absorber as an additional tool for vibration control of inverted pendulum structures is very limited in the literature. Anh et al. [21] used the stability optimization to give optimal parameters of a DVA for undamped inverted pendulum structures. To the best knowledge of the authors, there has been no study on the DVA using $H_{\infty}$ optimization for undamped structures and the DVA for damped inverted pendulum structures. Perhaps a reason is that the calculations in these cases are too complicated.

The equivalent linearization method is one of common approaches to approximate analysis of dynamic systems. The original linearization for deterministic systems was proposed by Krylov and Bogoliubov [28]. Afterwards, Caughey [29,30] expanded the method for stochastic systems. Thenceforth, there have been some extended versions of the equivalent linearization method. Recently, Anh et al. [31,32] proposed a so-called global-local criterion for the equivalent linearization method. In this paper, optimal analytical parameters of a DVA installed in an undamped inverted pendulum structure using $H_{\infty}$ optimization are presented. Afterwards, based on the idea of the global-local criterion, the authors suggest an analytical approach to give approximate analytical expressions of a DVA's parameters for damped structures by replacing the original damped structure by an equivalent undamped structure. Finally, numerical simulation is done for an example of the articulated tower in the ocean to validate the accuracy of the results obtained in this study.

\section{OPTIMAL PARAMETERS OF A DYNAMIC VIBRATION ABSORBER ATTACHED TO UNDAMPED INVERTED PENDULUM STRUCTURES}

Fig. 1 illustrates a pendulum type absorber attached to an undamped inverted pendulum structure. The primary structure has a concentrated mass $m$ at the top. The length of beam is $l$ and the length mass density is $\rho$. The torsion spring coefficient is $k_{s}$. A pendulum type absorber has the mass $m_{d}$, length $l_{d}$, spring constant $k_{d}$ and damping constant 
$c_{d}$. The absorber is installed in the main structure through a pulley with the radius $r$. The system is subjected to an external force $f(t)=f_{0} \sin \Omega t$.

Using Lagrange approach and ignoring the high power terms, the linear motion equations are written in the matrix form as follows [21]

$$
\begin{aligned}
& {\left[\begin{array}{cc}
m l^{2}+\frac{1}{3} \rho l^{3}+m_{d}\left(d-l_{d}\right)^{2} & m_{d} d l_{d}-m_{d} l_{d}^{2} \\
m_{d} d l_{d}-m_{d} l_{d}^{2} & m_{d} l_{d}^{2}
\end{array}\right]\left[\begin{array}{c}
\ddot{\theta} \\
\ddot{\theta}_{d}
\end{array}\right]+\left[\begin{array}{cc}
0 & 0 \\
0 & c_{d} r^{2}
\end{array}\right]\left[\begin{array}{c}
\dot{\theta} \\
\dot{\theta}_{d}
\end{array}\right]+} \\
& +\left[\begin{array}{cc}
k_{s}-m g l-\frac{1}{2} \rho g l^{2}-m_{d} g\left(d-l_{d}\right) & -m_{d} g l_{d} \\
-m_{d} g l_{d} & k_{d} r^{2}+m_{d} g l_{d}
\end{array}\right]\left[\begin{array}{c}
\theta \\
\theta_{d}
\end{array}\right]=\left[\begin{array}{c}
l f(t) \\
0
\end{array}\right] .
\end{aligned}
$$

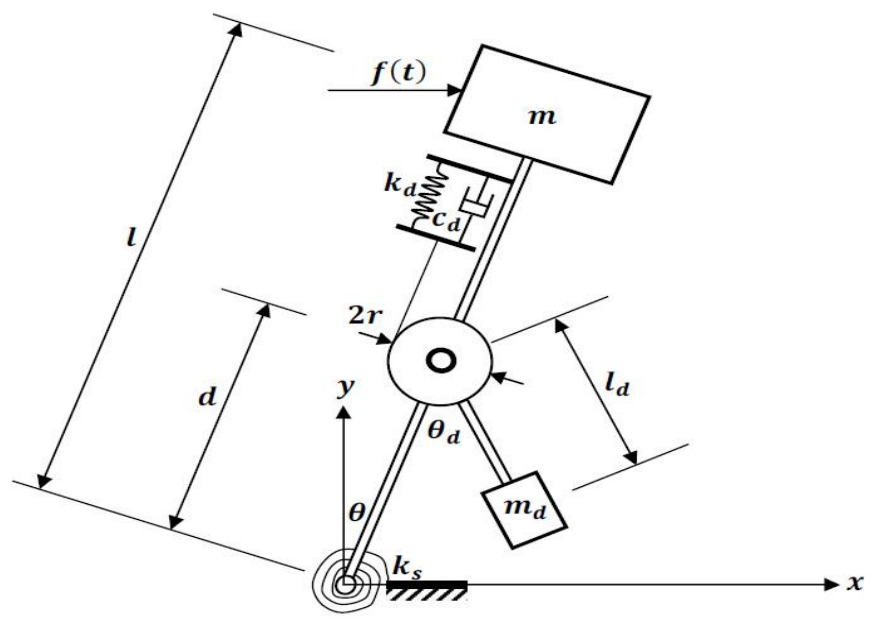

Fig. 1. A dynamic vibration absorber attached to an undamped inverted pendulum structure

We introduce

$$
\begin{aligned}
& \mu=\frac{m_{d}}{m+\frac{1}{3} \rho l}, \quad \gamma=\frac{d-l_{d}}{l}, \quad \omega_{s}=\sqrt{\frac{6 k_{s}-g l(6 m+3 \rho l)}{2 l^{2}(3 m+\rho l)}}, \\
& \omega_{d}=\sqrt{\frac{k_{d} r^{2}}{m_{d} l_{d}^{2}}+\frac{g}{l_{d}}}, \quad \alpha=\frac{\omega_{d}}{\omega_{s}}, \quad \xi_{d}=\frac{c_{d} r^{2}}{2 m_{d} \omega_{d} l_{d}^{2}}, \eta=\frac{g}{l \omega_{s}^{2}}, \quad \beta=\frac{\Omega}{\omega_{s}}, \\
& u=l \theta, \quad u_{d}=l_{d} \theta_{d},
\end{aligned}
$$

where $\mu$ is the mass ratio of the DVA to the main structure, $\gamma$ is the DVA's position, $\omega_{s}$ is the natural frequency of the main structure, $\omega_{d}$ and $\xi_{d}$ are the natural frequency and damping ratio of the DVA, respectively, $\alpha$ is the tuning ratio, $\eta$ specifies the mass distribution of the main structure, $\beta$ is the frequency ratio of the external force.

The matrix equation (1) can be rewritten as [21]

$$
\left[\begin{array}{cc}
1+\mu \gamma^{2} & \mu \gamma \\
\mu \gamma & \mu
\end{array}\right]\left[\begin{array}{c}
\ddot{u} \\
\ddot{u}_{d}
\end{array}\right]+\omega_{s}\left[\begin{array}{cc}
0 & 0 \\
0 & 2 \mu \alpha \xi_{d}
\end{array}\right]\left[\begin{array}{c}
\dot{u} \\
\dot{u}_{d}
\end{array}\right]+\omega_{s}^{2}\left[\begin{array}{cc}
1-\mu \gamma \eta & -\mu \eta \\
-\mu \eta & \mu \alpha^{2}
\end{array}\right]\left[\begin{array}{c}
u \\
u_{d}
\end{array}\right]=\left[\begin{array}{c}
3 f(t) /(3 m+\rho l) \\
0
\end{array}\right] .
$$




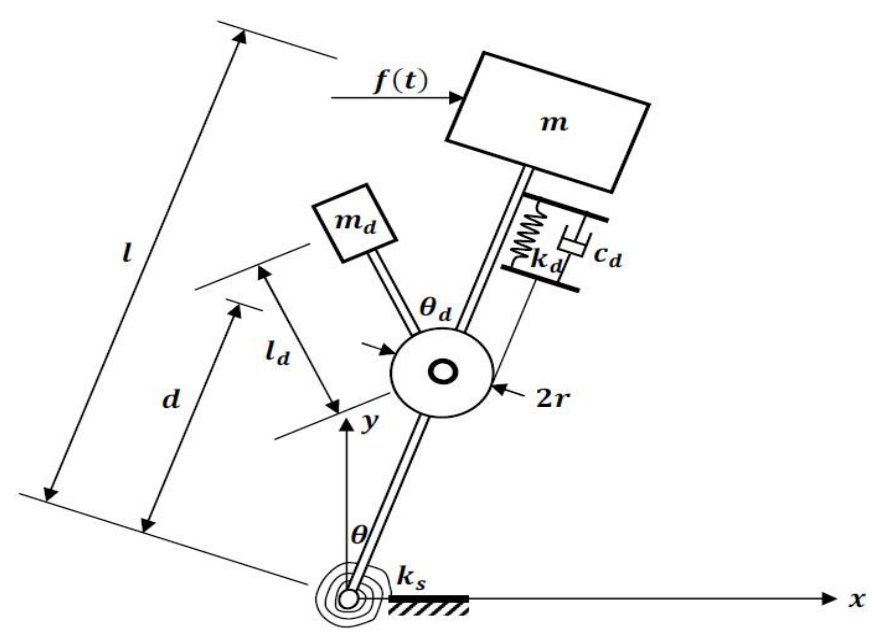

Fig. 2. An inverted pendulum type dynamic vibration absorber

As can be seen in Eq. (3), when $\eta=-1$, we obtain the conventional pendulum structures. When $\gamma=1$ and $\eta=0$, we have the mass-spring structures.

In many practical applications, the natural frequency of a DVA is required to be small. This means that the DVA's length is too long. To solve this problem, an inverted pendulum type DVA as shown in Fig. 2 is a good solution. In this case, the motion equations are similar to Eqs. (1) and (3) but in Eq. (2) we replace $l_{d}$ by $-l_{d}$. Anh et al. [21] have shown that the inverted pendulum DVA in Fig. 2 has better performance than the conventional pendulum DVA does.

To the best knowledge of the authors, there has been no study on a DVA using $H_{\infty}$ optimization. In this section, we will use the fixed-point method to give the optimal parameters of a DVA for the $H_{\infty}$ optimization. Using Eq. (3), the amplitude magnification factor of the primary structure is determined as follows

$$
H=\left|\frac{u}{3 f_{0} /(3 m+\rho l) \omega_{s}^{2}}\right|=\sqrt{\frac{H_{1}^{2}+H_{2}^{2} \xi_{d}^{2}}{H_{3}^{2}+H_{4}^{2} \xi_{d}^{2}}},
$$

where

$$
\begin{aligned}
& H_{1}=\alpha^{2}-\beta^{2}, \\
& H_{2}=2 \alpha \beta, \\
& H_{3}=\alpha^{2}(1-\mu \gamma \eta)-\mu \eta^{2}-\left(1+\alpha^{2}+\mu \alpha^{2} \gamma^{2}+\mu \gamma \eta\right) \beta^{2}+\beta^{4}, \\
& H_{4}=2 \alpha \beta\left(1-\mu \gamma \eta-\beta^{2}-\mu \gamma^{2} \beta^{2}\right) .
\end{aligned}
$$

Fig. 3 presents the graphs of the amplitude magnification factor $H$ versus the frequency ratio $\beta$ corresponding to some different values of the DVA's damping ratio $\xi_{d}$. We observe from this graphs that there exist two fixed points $P$ and $Q$ which are independent of $\xi_{d}$. At the first step of the fixed-point method, we will find two positions of $P$ and $Q$. Because $P$ and $Q$ are intersection points of the graphs $H$, the positions of $P$ and $Q$ can be 


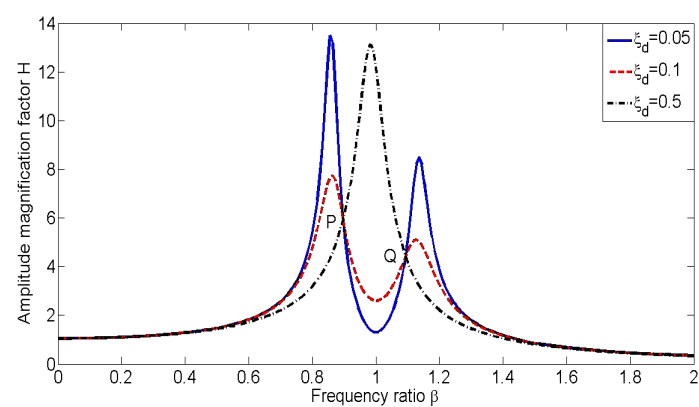

Fig. 3. Graphs of the amplitude magnification factor versus the frequency ratio $\beta$



Fig. 4. A comparison of the amplitude magnification factor $H$

determined by letting $\xi_{d}=0$ and $\xi_{d}=\infty$ in Eq. (4). We obtain

$$
\left|\frac{H_{1}}{H_{3}}\right|=\left|\frac{H_{2}}{H_{4}}\right| \text {. }
$$

Substituting Eq. (5) into Eq. (6) leads to an equation for $\beta_{P}$ and $\beta_{Q}$ as

$$
\left(2+\mu \gamma^{2}\right) \beta^{4}-2\left(1+\alpha^{2}+\mu \gamma^{2} \alpha^{2}\right) \beta^{2}+2 \alpha^{2}(1-\mu \gamma \eta)-\mu \eta^{2}=0 .
$$

At the second step, the highs of $P$ and $Q$ are adjusted to be equal, i.e. we have

$$
\left|\frac{H_{2}}{H_{4}}\right|\left(\beta_{P}\right)=\left|\frac{H_{2}}{H_{4}}\right|\left(\beta_{Q}\right) .
$$

Combining Eq. (7) with Eq. (8), we obtain an optimal value of the tuning ratio as follows

$$
\alpha=\frac{\sqrt{1-\mu \gamma \eta\left(2+\mu \gamma^{2}\right)}}{1+\mu \gamma^{2}}
$$

At the third step, the DVA's damping ratio $\xi_{d}$ is determined by letting $P$ and $Q$ be two peaks of $H$, namely

$$
\frac{\partial H}{\partial \beta}\left(\beta_{P}\right)=\frac{\partial H}{\partial \beta}\left(\beta_{Q}\right)=0 .
$$

Using Eqs. (4) and (10) yields

$$
\xi_{d}(P)=\frac{H^{2} H_{3} \frac{\partial H_{3}}{\partial \beta}-H_{1} \frac{\partial H_{1}}{\partial \beta}}{H_{2} \frac{\partial H_{2}}{\partial \beta}-H^{2} H_{4} \frac{\partial H_{4}}{\partial \beta}}\left(\beta_{P}\right), \quad \xi_{d}(Q)=\frac{H^{2} H_{3} \frac{\partial H_{3}}{\partial \beta}-H_{1} \frac{\partial H_{1}}{\partial \beta}}{H_{2} \frac{\partial H_{2}}{\partial \beta}-H^{2} H_{4} \frac{\partial H_{4}}{\partial \beta}}\left(\beta_{Q}\right) .
$$

The optimal damping ratio of the DVA are found as

$$
\xi_{d}=\sqrt{\frac{\xi_{d}^{2}(P)+\xi_{d}^{2}(Q)}{2}} .
$$


Introducing Eqs. (4) and (5) into Eq. (11), using Eqs. (7) and (12), we obtain

$$
\xi_{d}=\frac{\sqrt{\frac{1-\alpha^{2}\left(3+\mu \gamma^{2}\right)}{2+\mu \gamma^{2}}+\frac{\alpha^{4}\left(2+\mu \gamma^{2}+\mu^{2} \gamma^{3} \eta\right)+\alpha^{2}\left(2 \mu \gamma \eta+\mu \eta^{2}+\mu^{2} \gamma^{2} \eta^{2}\right)+\mu \eta^{2}}{2 \alpha^{2}(1-\mu \gamma \eta)-\mu \eta^{2}}}}{2 \alpha \sqrt{1+\mu \gamma^{2}}} .
$$

Finally, combining Eq. (9) with Eq. (13), we have

$$
\begin{aligned}
\alpha & =\frac{\sqrt{1-\mu \gamma \eta\left(2+\mu \gamma^{2}\right)}}{1+\mu \gamma^{2}}, \\
\xi_{d} & =\frac{\sqrt{\frac{1-\alpha^{2}\left(3+\mu \gamma^{2}\right)}{2+\mu \gamma^{2}}+\frac{\alpha^{4}\left(2+\mu \gamma^{2}+\mu^{2} \gamma^{3} \eta\right)+\alpha^{2}\left(2 \mu \gamma \eta+\mu \eta^{2}+\mu^{2} \gamma^{2} \eta^{2}\right)+\mu \eta^{2}}{2 \alpha^{2}(1-\mu \gamma \eta)-\mu \eta^{2}}}}{2 \alpha \sqrt{1+\mu \gamma^{2}}} .
\end{aligned}
$$

The results (14) are two optimal parameters of a pendulum type DVA in the case of undamped inverted pendulum structures. These results will reduce to Den Hartog's results [3] for mass-spring structures when $\gamma=1, \eta=0$ and to Matsuhisa et al.'s results [17] for conventional pendulum structures when $\eta=-1$. Fig. 4 shows the DVA's effectiveness when it is installed in a primary structure. It is seen that the DVA has a great performance in vibration reduction of the primary structure. Furthermore, the optimal DVA designed by Eq. (14) makes the peak of the amplitude magnification factor $\mathrm{H}$ considerably smaller than an un-optimal DVA does. However, the analytical solution in Eq. (14) requires an extension to damped structures because the damping always exists in real structures. In the following sections, a simple approach will be presented to design a DVA for damped inverted pendulum structures.

\section{AN EQUIVALENT UNDAMPED STRUCTURE}

In this section, we will use the global-local approach presented in Ref. [32] in order to replace approximately the original damped structure by an equivalent undamped structure as shown in Fig. 5.

In the case of Fig. 5a with damped original structure, to the first order the equation of motion is given as follows

$$
\ddot{\theta}+2 \xi_{s} \omega_{s} \dot{\theta}+\omega_{s}^{2} \theta=0 .
$$

In order to obtain the equivalent undamped structure (see Fig. $5 b$ ) we replace the viscous element $2 \xi_{s} \omega_{s} \dot{\theta}$ by the spring term $\gamma \theta$

$$
2 \xi_{s} \omega_{s} \dot{\theta} \approx \gamma \theta,
$$

where $\gamma$ is the equivalent coefficient that will be determined by the global-local criterion. Based on Eq. (16), the damped system 5a is approximately replaced by the undamped system $5 \mathrm{~b}$ and the equation (15) becomes as follows

$$
\ddot{\theta}+\omega_{e}^{2} \theta=0,
$$



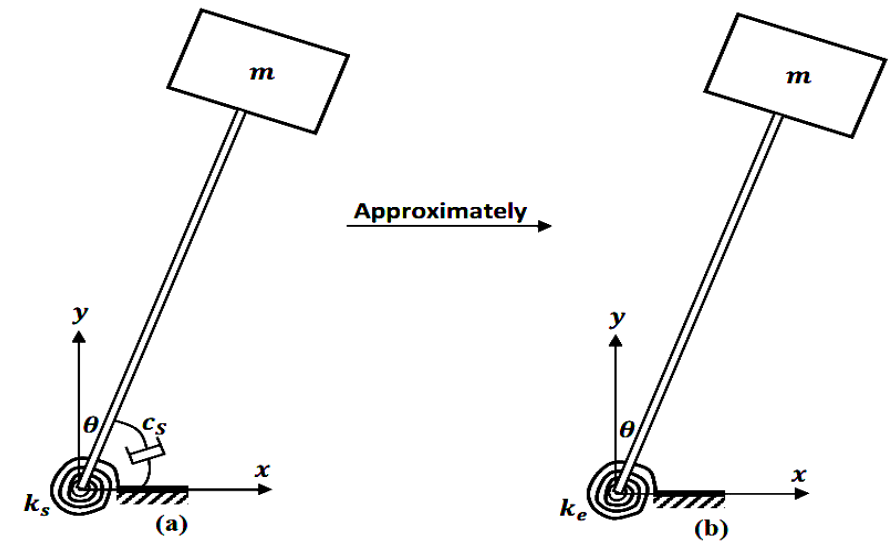

Fig. 5. An approximation of primary structure

where $\omega_{e}$ is the equivalent frequency

$$
\omega_{e}^{2}=\omega_{s}^{2}+\gamma
$$

Eq. (17) gives

$$
\theta=\Theta \cos \varphi, \quad \dot{\theta}=-\omega_{e} \Theta \sin \varphi, \quad \varphi=\omega_{e} t+\varphi_{0} .
$$

Using the global-local criterion at local step we have

$$
S=\left\langle\left(2 \xi_{s} \omega_{s} \dot{\theta}-\gamma \theta\right)^{2}\right\rangle_{\Phi} \rightarrow \min _{\gamma}
$$

where the local averaging operator

$$
\langle.\rangle_{\Phi}=\frac{1}{\Phi} \int_{0}^{\Phi}(.) \mathrm{d} \phi,
$$

is introduced for a local domain $\Phi$ with $0 \leq \Phi \leq 2 \pi$.

The criterion (20) leads to $\mathrm{d} S / \mathrm{d} \gamma=\overline{0}$. Thus, we have

$$
\left\langle\left(2 \xi_{s} \omega_{s} \dot{\theta}-\gamma \theta\right) \theta\right\rangle_{\Phi}=0,
$$

or

$$
\gamma=2 \xi_{s} \omega_{s} \frac{\langle\theta \dot{\theta}\rangle_{\Phi}}{\left\langle\theta^{2}\right\rangle_{\Phi}}
$$

Combining Eqs. (19) and (21), we get

$$
\begin{aligned}
& \langle\theta \dot{\theta}\rangle_{\Phi}=-\frac{\Theta^{2} \omega_{e} \sin ^{2} \Phi}{2 \Phi} \\
& \left\langle\theta^{2}\right\rangle_{\Phi}=\frac{\Theta^{2}}{4 \Phi}(2 \Phi+\sin 2 \Phi) .
\end{aligned}
$$


Substituting Eqs. (24) into Eq. (23) yields

$$
\gamma=-4 \xi_{s} \omega_{s} \omega_{e} \frac{\sin ^{2} \Phi}{2 \Phi+\sin 2 \Phi}
$$

Using Eqs. (18) and (25), we obtain

$$
\omega_{e}^{2}+4 \xi_{s} \omega_{s} \omega_{e} \frac{\sin ^{2} \Phi}{2 \Phi+\sin 2 \Phi}-\omega_{s}^{2}=0 .
$$

Eq. (26) is a quadratic equation in terms of frequency $\omega_{e}$. Solving this equation leads to

$$
\omega_{e}(\Phi)=\omega_{s}\left[\sqrt{1+\frac{4 \sin ^{4} \Phi \xi_{s}^{2}}{(2 \Phi+\sin 2 \Phi)^{2}}}-\frac{2 \sin ^{2} \Phi}{2 \Phi+\sin 2 \Phi} \xi_{s}\right]
$$

At the global step, the equivalent frequency $\omega_{e}$ is finally determined as the average value of all local equivalent frequencies as follows

$$
\omega_{e}=\frac{1}{2 \pi} \int_{0}^{2 \pi} \omega_{e}(\Phi) \mathrm{d} \Phi=\frac{1}{2 \pi} \int_{0}^{2 \pi} \omega_{s}\left[\sqrt{1+\frac{4 \sin ^{4} \Phi \xi_{s}^{2}}{(2 \Phi+\sin 2 \Phi)^{2}}}-\frac{2 \sin ^{2} \Phi}{2 \Phi+\sin 2 \Phi} \xi_{s}\right] \mathrm{d} \Phi .
$$

We have replaced the damped primary structure with an equivalent undamped structure where the approximate frequency $\omega_{e}$ is given in Eq. (28). In section 4, we will use the above results to give the approximate optimal parameters for a DVA attached to damped inverted pendulum structures.

\section{DYNAMIC VIBRATION ABSORBER FOR DAMPED STRUCTURES}

Fig. 6 illustrates a system consisting of a pendulum type DVA and a damped inverted pendulum structure. The motion equations for this case are

$$
\left[\begin{array}{cc}
1+\mu \gamma^{2} & \mu \gamma \\
\mu \gamma & \mu
\end{array}\right]\left[\begin{array}{c}
\ddot{u} \\
\ddot{u}_{d}
\end{array}\right]+\omega_{s}\left[\begin{array}{cc}
2 \xi_{s} & 0 \\
0 & 2 \mu \alpha \xi_{d}
\end{array}\right]\left[\begin{array}{c}
\dot{u} \\
\dot{u}_{d}
\end{array}\right]+\omega_{s}^{2}\left[\begin{array}{cc}
1-\mu \gamma \eta & -\mu \eta \\
-\mu \eta & \mu \alpha^{2}
\end{array}\right]\left[\begin{array}{c}
u \\
u_{d}
\end{array}\right]=\left[\begin{array}{c}
3 f(t) /(3 m+\rho l) \\
0
\end{array}\right] .
$$

The amplitude magnification factor is determined as follows

$$
H=\left|\frac{u}{3 f_{0} /(3 m+\rho l) \omega_{s}^{2}}\right|=\sqrt{\frac{A^{2}+B^{2}}{C^{2}+D^{2}}},
$$

in which

$$
\begin{aligned}
& A=\alpha^{2}-\beta^{2}, \\
& B=2 \alpha \beta \xi_{d}, \\
& C=\alpha^{2}(1-\mu \gamma \eta)-\mu \eta^{2}-\left(1+\alpha^{2}+\mu \alpha^{2} \gamma^{2}+\mu \gamma \eta+4 \alpha \xi_{s} \xi_{d}\right) \beta^{2}+\beta^{4}, \\
& D=2 \alpha \beta \xi_{d}\left(1-\mu \gamma \eta-\beta^{2}-\mu \gamma^{2} \beta^{2}\right)+2 \xi_{s} \beta\left(\alpha^{2}-\beta^{2}\right) .
\end{aligned}
$$

Using the results (14) for the equivalent undamped structure, we have 


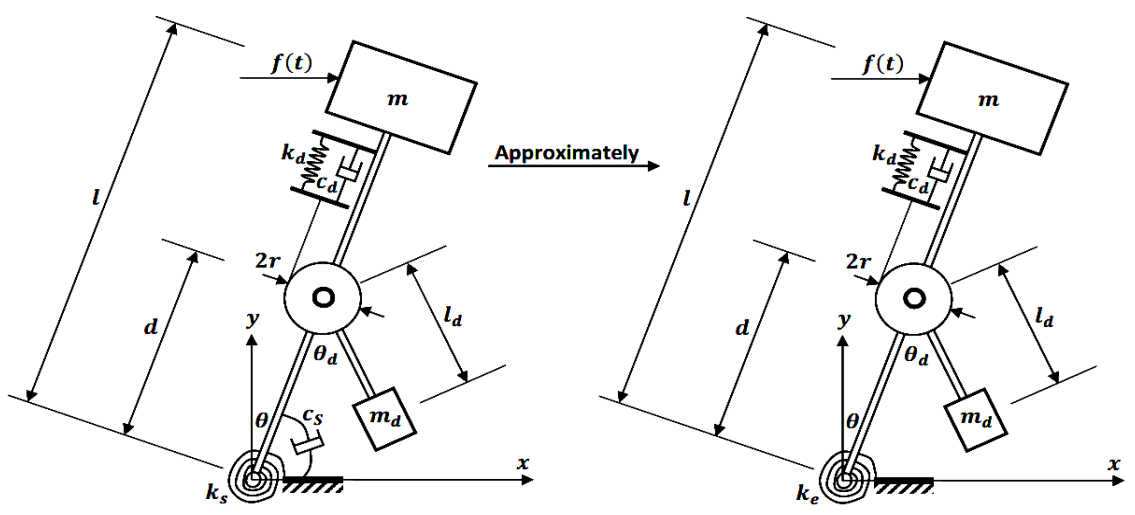

Fig. 6. A pendulum type DVA installed in a damped inverted pendulum structure and an equivalent system

$$
\begin{aligned}
& \alpha_{e}=\frac{\sqrt{1-\mu \gamma \eta\left(2+\mu \gamma^{2}\right)}}{1+\mu \gamma^{2}}, \\
& \xi_{d e}=\frac{\sqrt{\frac{1-\alpha_{e}^{2}\left(3+\mu \gamma^{2}\right)}{2+\mu \gamma^{2}}+\frac{\alpha_{e}^{4}\left(2+\mu \gamma^{2}+\mu^{2} \gamma^{3} \eta\right)+\alpha_{e}^{2}\left(2 \mu \gamma \eta+\mu \eta^{2}+\mu^{2} \gamma^{2} \eta^{2}\right)+\mu \eta^{2}}{2 \alpha_{e}^{2}(1-\mu \gamma \eta)-\mu \eta^{2}}}}{2 \alpha_{e} \sqrt{1+\mu \gamma^{2}}} .
\end{aligned}
$$

It is noted that

$$
\alpha_{e}=\frac{\omega_{d}}{\omega_{e}} ; \alpha=\frac{\omega_{d}}{\omega_{s}} .
$$

Using Eqs. (28), (32) and (33), we obtain the DVA's parameters as follows

$$
\begin{aligned}
& \alpha=\frac{\sqrt{1-\mu \gamma \eta\left(2+\mu \gamma^{2}\right)}}{2 \pi\left(1+\mu \gamma^{2}\right)} \int_{0}^{2 \pi}\left[\sqrt{1+\frac{4 \sin ^{4} \Phi \xi_{s}^{2}}{(2 \Phi+\sin 2 \Phi)^{2}}}-\frac{2 \sin ^{2} \Phi}{2 \Phi+\sin 2 \Phi} \xi_{s}\right] \mathrm{d} \Phi, \\
& \xi_{d}=\frac{\sqrt{\frac{1-\alpha^{2}\left(3+\mu \gamma^{2}\right)}{2+\mu \gamma^{2}}+\frac{\alpha^{4}\left(2+\mu \gamma^{2}+\mu^{2} \gamma^{3} \eta\right)+\alpha^{2}\left(2 \mu \gamma \eta+\mu \eta^{2}+\mu^{2} \gamma^{2} \eta^{2}\right)+\mu \eta^{2}}{2 \alpha^{2}(1-\mu \gamma \eta)-\mu \eta^{2}}}}{2 \alpha \sqrt{1+\mu \gamma^{2}}} .
\end{aligned}
$$

Eq. (34) is an approximate analytical solution for optimal parameters of a DVA attached to a damped inverted pendulum structure. The effectiveness of these results will be validated in next section.

\section{VALIDATION}

\subsection{Undamped inverted primary pendulum structures}

In this section, the result (14) proposed in this paper is compared with the solution given by Ref. [21] as follows 


$$
\begin{aligned}
& \alpha_{\text {Ref.[21] }}=\frac{\sqrt{(1-\gamma \eta \mu)^{2}+\mu \eta^{2}\left(1+\gamma^{2} \mu\right)^{2}}}{\left(1+\gamma^{2} \mu\right) \sqrt{1-\gamma \eta \mu}}, \\
& \xi_{\text {d_Ref.[21] }}=\frac{\sqrt{\mu(\gamma+\eta)^{2}}}{\sqrt{\left(1+\gamma^{2} \mu\right)\left[(1-\gamma \eta \mu)^{2}+\mu \eta^{2}\left(1+\gamma^{2} \mu\right)^{2}\right]}} .
\end{aligned}
$$

Figs. 7 and 8 describe the comparisons of the amplitude magnification factor $H$. The solid line is the graph when using Eq. (14) and the dashed line is the case using the result given by Ref. [21]. As we can observe, the peak of the amplitude magnification factor is considerably lowered when using the DVA designing from Eq. (14) suggested in this paper.

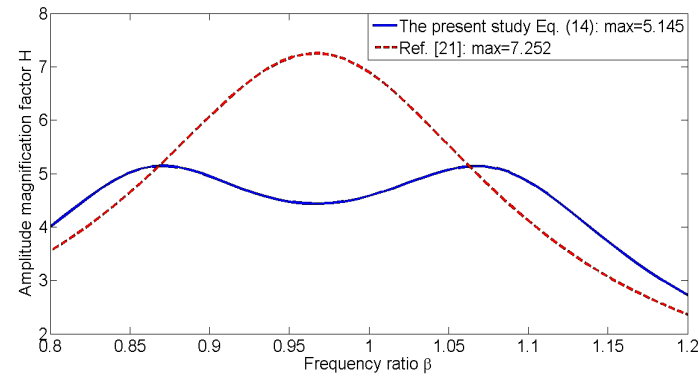

Fig. 7. Comparison of the amplitude magnification factors $H$ for undamped primary structures where $\mu=0.03, \gamma=1, \eta=0.6$

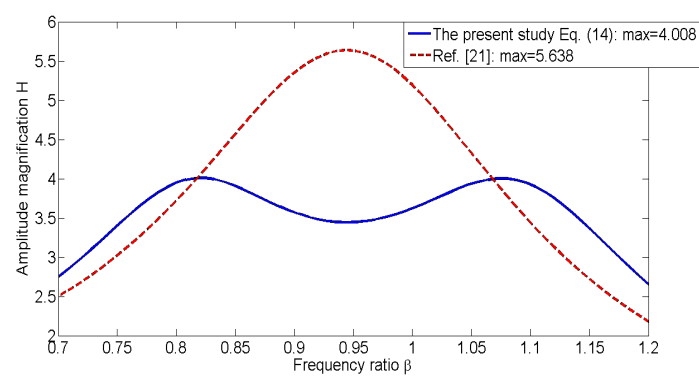

Fig. 8. Comparison of the amplitude magnification factors $H$ for undamped primary structures where $\mu=0.05, \gamma=1, \eta=0.6$

\subsection{Damped inverted primary pendulum structures}

Comparisons of Eq. (14) with Eq. (34) for damped inverted primary pendulum structures are illustrated in Figs. 9, 10, 11 and 12. We can see that the peaks of the

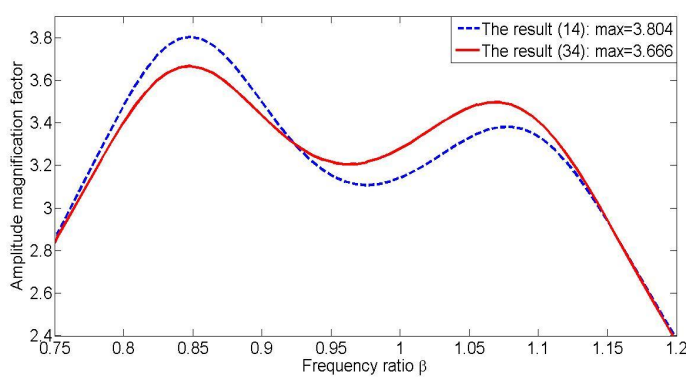

Fig. 9. Comparison of the amplitude magnification factors $H$ for damped primary structures where $\mu=0.03, \gamma=1, \eta=0.6, \xi_{s}=0.05$

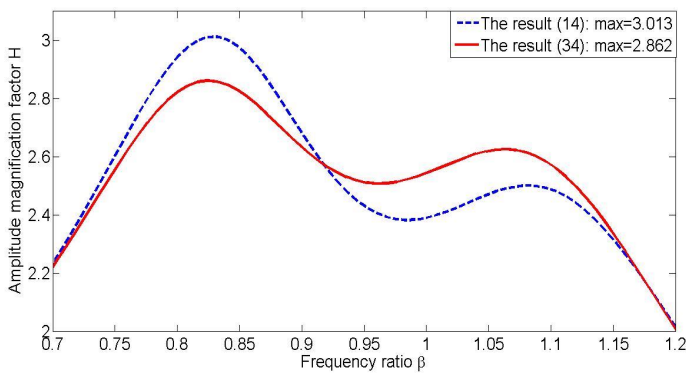

Fig. 10. Comparison of the amplitude magnification factors $H$ for damped primary structures where $\mu=0.03, \gamma=1, \eta=0.6, \xi_{s}=0.1$ 




Fig. 11. Comparison of the amplitude magnification factors $H$ for damped primary structures where $\mu=0.05, \gamma=1, \eta=0.6, \xi_{s}=0.05$

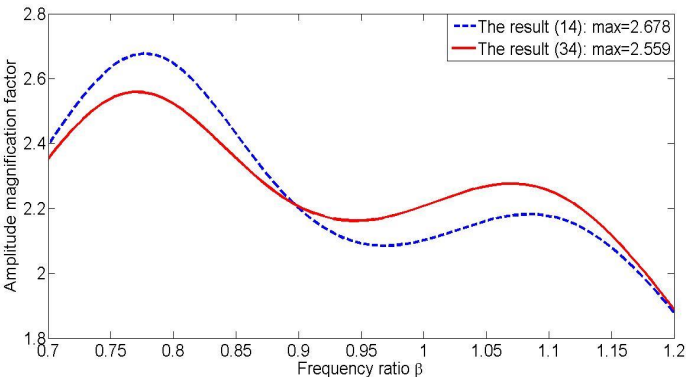

Fig. 12. Comparison of the amplitude magnification factors $H$ for damped primary structures where $\mu=0.05, \gamma=1, \eta=0.6, \xi_{s}=0.1$

amplitude magnification factor $H$ using the result (34) are significantly lower than those using the solution (14). Therefore, the result (34) for damped structures proposed in this study is useful in practice.

\section{CONCLUSIONS}

This paper is concerned with an optimization problem of a pendulum type dynamic vibration absorber for inverted pendulum structures. This model is a general case where the conventional pendulum and mass-spring structures can be obtained from it as special cases. In practice, the inverted pendulum can be used to model many real structures as the arms of robots, soil structures, or fluid structures. To the best knowledge of the authors, there has been no study on the design of a DVA for undamped inverted pendulum structures using $H_{\infty}$ optimization as well as a DVA for damped structures. The novelty of this study can be summarized below.

- Optimal parameters of a DVA attached to an undamped inverted pendulum structures using $H_{\infty}$ are found as in Eq. (14).

- Approximate analytical expressions of a DVA's optimal parameters are given for damped structures as shown in Eq. (34). The main idea is using the global-local approach to replace approximately the damped primary structure with an equivalent undamped structure. After that, the parameters of a DVA are obtained by using known results for undamped structures.

- Comparisons are done to show that the results suggested in this paper are useful in practice.

\section{ACKNOWLEDGEMENTS}

This paper is funded by Vietnam National Foundation for Science and Technology Development (NAFOSTED) under grant number: 107.04-2013.19.

\section{REFERENCES}

[1] H. Frahm. Device for damping vibrations of bodies, (30 October, 1909). U.S. Patent No. 989958. 
[2] J. Ormondroyd and J. P. Den Hartog. Theory of the dynamic vibration absorber. Transaction of the ASME, Journal of Applied Mechanics, 50, (7), (1928), pp. 9-22.

[3] J. Den Hartog. Mechanical vibrations. McGraw-Hill, New York, (1956).

[4] O. Nishihara and T. Asami. Closed-form solutions to the exact optimizations of dynamic vibration absorbers (minimizations of the maximum amplitude magnification factors). Journal of Vibration and Acoustics, 124, (4), (2002), pp. 576-582.

[5] S. H. Crandall and W. D. Mark. Random vibration in mechanical systems. Academic Press, (1963).

[6] Y. Iwata. On the construction of the dynamic vibration absorbers. Preparation of the Japan Society of Mechanical Engineering, 820, (8), (1982), pp. 150-152. (in Japanese).

[7] T. Asami, T. Wakasono, K. Kameoka, M. Hasegawa, and H. Sekiguchi. Optimum design of dynamic absorbers for a system subjected to random excitation. JSME International Journal, Series 3, Vibration, Control Engineering, Engineering for Industry, 34, (2), (1991), pp. 218-226.

[8] H. Yamaguchi. damping of transient vibration by a dynamic absorber. Transactions of the Japan Society of Mechanical Engineering, Series C, 54, (499), (1988), pp. 561-568. (in Japanese).

[9] T. Ioi and K. Ikeda. On the dynamic vibration damped absorber of the vibration system. Bulletin of Bulletin of the Japanese Society of Mechanical Engineering, 21, (151), (1978), pp. 64-71.

[10] E. Pennestri. An application of Chebyshev's min-max criterion to the optimal design of a damped dynamic vibration absorber. Journal of Sound and Vibration, 217, (4), (1998), pp. 757765.

[11] T. Asami, O. Nishihara, and A. M. Baz. Analytical solutions to $\mathrm{H} \infty$ and $\mathrm{H}_{2}$ optimization of dynamic vibration absorbers attached to damped linear systems. Journal of Vibration and Acoustics, 124, (2), (2002), pp. 284-295.

[12] A. Ghosh and B. Basu. A closed-form optimal tuning criterion for TMD in damped structures. Structural Control and Health Monitoring, 14, (4), (2007), pp. 681-692.

[13] N. D. Anh and N. X. Nguyen. Extension of equivalent linearization method to design of TMD for linear damped systems. Structural Control and Health Monitoring, 19, (6), (2012), pp. 565573.

[14] N. D. Anh and N. X. Nguyen. Design of TMD for damped linear structures using the dual criterion of equivalent linearization method. International Journal of Mechanical Sciences, 77, (2013), pp. 164-170.

[15] O. F. Tigli. Optimum vibration absorber (tuned mass damper) design for linear damped systems subjected to random loads. Journal of Sound and Vibration, 331, (13), (2012), pp. 30353049 .

[16] O. Nishihara and H. Matsuhisa. Design and tuning of vibration control devices via stability criterion. Preparation of the Japan Society of Mechanical Engineering, 10, (1), (1997), pp. 165-168.

[17] H. Matsuhisa, R. Gu, Y. Wang, O. Nishihara, and S. Sato. Vibration control of a ropeway carrier by passive dynamic vibration absorbers. JSME International Journal, Serires $C$, Dynamics, Control, Robotics, Design and Manufacturing, 38, (4), (1995), pp. 657-662.

[18] L. D. Viet, N. D. Anh, and H. Matsuhisa. The effective damping approach to design a dynamic vibration absorber using Coriolis force. Journal of Sound and Vibration, 330, (9), (2011), pp. 1904-1916.

[19] L. D. Viet, N. D. Anh, and H. Matsuhisa. Vibration control of a pendulum structure by a dynamic vibration absorber moving in both normal and tangential directions. Proceedings of the Institution of Mechanical Engineers, Part C: Journal of Mechanical Engineering Science, 225, (5), (2011), pp. 1087-1095. 
[20] L. D. Viet. Sequential design of two orthogonal dynamic vibration absorbers in a pendulum based on stability maximization. Proceedings of the Institution of Mechanical Engineers, Part C: Journal of Mechanical Engineering Science, 226, (2012), pp. 2645-2655.

[21] N. D. Anh, H. Matsuhisa, L. D. Viet, and M. Yasuda. Vibration control of an inverted pendulum type structure by passive mass-spring-pendulum dynamic vibration absorber. Journal of Sound and Vibration, 307, (1), (2007), pp. 187-201.

[22] W. H. Wu. Equivalent fixed-base models for soil-structure interaction systems. Soil Dynamics and Earthquake Engineering, 16, (5), (1997), pp. 323-336.

[23] P. Dong, H. Benaroya, and T. Wei. Integrating experiments into an energy-based reducedorder model for vortex-induced-vibrations of a cylinder mounted as an inverted pendulum. Journal of Sound and Vibration, 276, (1), (2004), pp. 45-63.

[24] P. Bar-Avi and H. Benaroya. Non-linear dynamics of an articulated tower in the ocean. Journal of Sound and Vibration, 190, (1), (1996), pp. 77-103.

[25] P. Bar-Avi and H. Benaroya. Stochastic response of a two DOF articulated tower. International Journal of Non-linear Mechanics, 32, (4), (1997), pp. 639-655.

[26] S. K. Chakrabarti and D. C. Cotter. Motion analysis of articulated tower. Journal of the Waterway Port Coastal and Ocean Division, 105, (3), (1979), pp. 281-292.

[27] O. Gottlieb, S. C. S. Yim, R. T. Hudspeth, et al. Analysis of nonlinear response of an articulated tower. International Journal of Offshore and Polar Engineering, 2, (1), (1992), pp. 61-66.

[28] N. M. Krylov and N. N. Bogoliubov. Introduction to non-linear mechanics. Princeton University Press, (1943).

[29] T. Caughey. Response of Van der Pol's oscillator to random excitation. Transactions of ASME, Journal of Applied Mechanics, 26, (3), (1959), pp. 345-348.

[30] T. Caughey. Random excitation of a system with bilinear hysteresis. Transactions of ASME, Journal of Applied Mechanics, 27, (4), (1960), pp. 649-652.

[31] N. Anh, L. Hung, and L. Viet. Dual approach to local mean square error criterion for stochastic equivalent linearization. Acta Mechanica, 224, (2), (2013), pp. 241-253.

[32] N. D. Anh and N. X. Nguyen. Global-local approach to the design of dynamic vibration absorber for damped structures. Journal of Vibration and Control, (2014). DOI: $10.1177 / 1077546314561282$. 